\title{
Fostering Research Synergies between Chemists in Swiss Academia and at Novartis
}

\author{
Arndt Meyer, Daniel Baeschlin, Cara E. Brocklehurst, Myriam Duckely, Fabrice Gallou, Lucie E. \\ Lovelle, Michael Parmentier, Thierry Schlama, Radka Snajdrova, and Yves P. Auberson*
}

\begin{abstract}
We present a short overview of the way Novartis chemists interact and collaborate with the academic chemistry community in Switzerland. This article exemplifies a number of collaborations, and illustrates opportunities to foster research synergies between academic and industrial researchers. It also describes established programs available to academic groups, providing them access to Novartis resources and expertise.
\end{abstract}

Keywords: Chemical development $\cdot$ Chemistry $\cdot$ Collaborations $\cdot$ Drug discovery $\cdot$ Partnerships

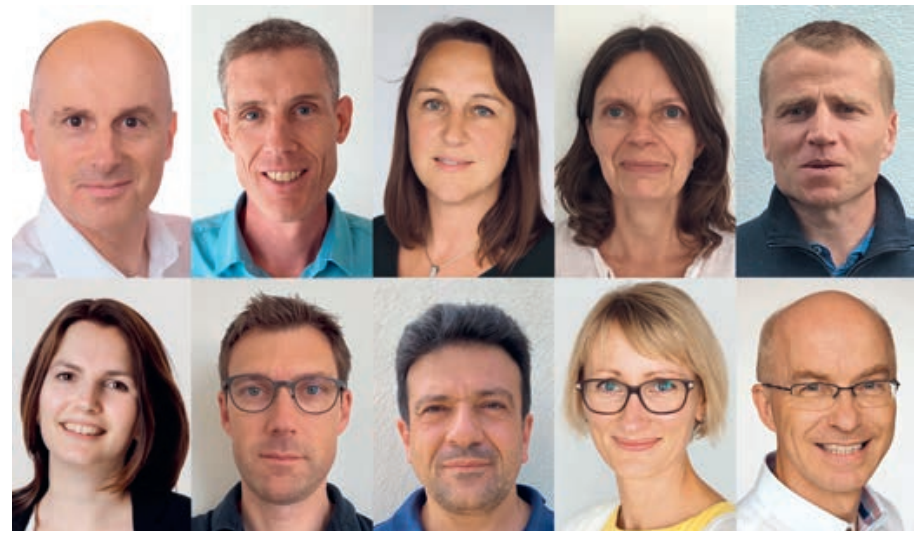

This mosaic portrays the authors of the manuscript. All are involved in chemistry drug discovery and development projects, collaborating across research and chemical development, and are located on the Novartis campus in Basel, Switzerland. From left to right, first row: Arndt Meyer, Daniel Baeschlin, Cara E. Brocklehurst, Myriam Duckely, Fabrice Gallou; second row: Lucie E. Lovelle, Michael Parmentier, Thierry Schlama, Radka Snajdrova, Yves P. Auberson.

\section{Introduction}

Interactions between Novartis chemists and Swiss academic partners take place at all stages of drug discovery and development. These include exploratory scientific partnerships with Novartis' chemistry research platform (GDC, Global Discovery Chemistry), technology projects with its chemical development unit (CHAD, Chemical and Analytical Development), and many community support activities. In parallel to the pursuit of scientific objectives, academic collaborators benefit from these interactions by gaining access to the Novartis infrastructure, chemical tools and expertise. Such interactions may also provide students with a first exposure to industrial research. In exchange, they allow Novartis chemists to explore novel concepts and technologies with academic partners, as well as to expand their scientific network. Successful partnerships achieve a defined scientific goal while mutually benefiting both parties.
In this article, we highlight a number of past and present projects, illustrating the opportunities for many types of collaborations. Some follow purely scientific interests while others are complementing Novartis research capabilities and enable projects of shared scientific value. As a matter of principle, we favour participative scientific partnerships, where both sides contribute at the same scientific level, with common goals and knowledge sharing. Most of the time these collaborations lead to publications in the scientific literature. In contrast, collaborations implying more limited creative contributions are run with contract research organizations, and are neither in the scope of this article nor of our interactions with academic groups.

Novartis chemists are involved in collaborations along the whole drug discovery, development and manufacturing process (Fig. 1). Some collaborations address specific questions related to target identification, drug discovery or chemical development. Others relate to the development of transformative methodologies, with the potential to influence multiple projects favourably, e.g. by enabling a seamless transition from research into development. More specifically, areas of interest at the early stages of drug discovery relate to chemical tool development for target validation, hit generation and drug candidate optimization, as well as accessing new chemical scaffolds. Interests related to chemical development often focus on new methods to increase synthetic efficiency and safety, reduce waste and energy demand, or address specific needs such as the reliance on transition metals of low natural abundance. Fig. 2 summarizes the overall collaboration opportunities in research and development.

While an exhaustive list of actual partnerships is beyond the scope of this article, we highlight some categories below.

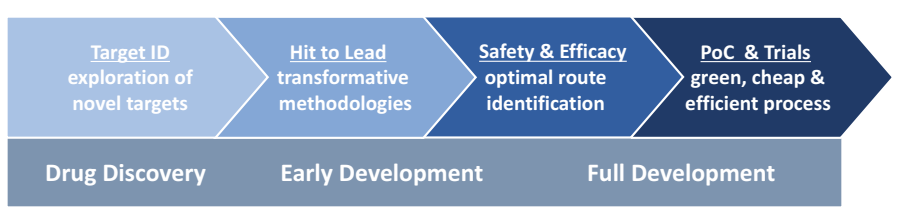

Fig. 1. Opportunities for academia-industry collaboration across research and development. PoC: Proof of Concept.

\section{Collaboration Examples}

\subsection{Developing Chemical Tools}

These collaborations aim to develop, characterize and use chemical probes to explore cellular biology or physiological pro- 


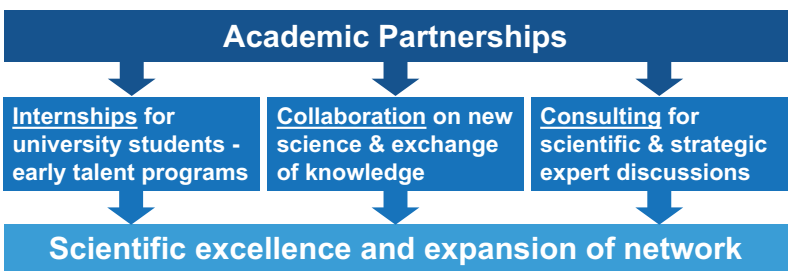

Fig. 2. External collaboration framework for chemical research at Novartis.

cesses. Ultimately, they address scientific questions by enabling in vitro or in vivo assays, taking advantage of the complementary expertise between academic and industrial partners. This enables both parties to join forces and to address questions that none could explore alone. Such an example is the development of specific chemical probes for imaging studies, based on fluorescence or radioactive isotopes.

\subsubsection{Developing Positron Emission Tomography (PET) Imaging Agents}

PET imaging agents play an important role in supporting the development of new drug candidates by providing a method to quantify the relationship between dose and target occupancy. This is particularly valuable for drugs acting in the brain, an organ that is difficult to sample directly. Our long-term collaboration with Prof. Simon Ametamey, at the Institute for Pharmaceutical Sciences of ETH Zurich, is a striking example of a long-term and fruitful partnership. It started in 1994, stoked by a mutual interest to develop PET imaging agents for glutamate receptors in the human brain. The partnership was a perfect fit from the beginning: Novartis was in a position to optimize and profile potential imaging tracers for several glutamate receptors but did not have the capacity to radiolabel them with ${ }^{11} \mathrm{C}$ or ${ }^{18} \mathrm{~F}$ and image their distribution in living organisms - an area in which Prof. Ametamey's group excels. Over time, several tracers were developed and published, including $\left[{ }^{11} \mathrm{C}\right] \mathrm{ABP} 688$ (Fig. 3), which is now broadly used for clinical imaging of metabotropic glutamate type five receptors (mGluR5). ${ }^{[1]}$
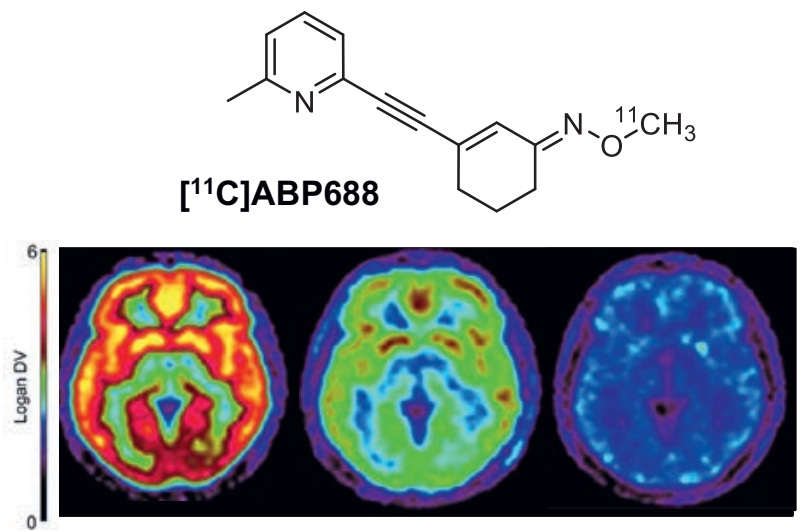

Fig. 3. Structure of $\left[{ }^{11} \mathrm{C}\right] \mathrm{ABP} 688$ and images from a clinical study with mavoglurant, showing dose-dependent target occupancy. Left: baseline; centre: $25 \mathrm{mg}$ dose; right: $200 \mathrm{mg}$ dose.

Much more recently, we explored a series of N-methyl-Daspartate (NMDA) receptor ligands specific for the GluN2A subunit, as potential imaging agents. While this particular project did not lead to a suitable clinical imaging agent, it allowed the discovery and publication of valuable chemical probes for the study of NMDA receptors. ${ }^{[2]}$

\subsection{Pre-competitive Research Initiatives}

Novartis also teams up with academic groups and other companies to share information and coordinate efforts, to explore questions of wider interest for the scientific community. Ultimately, this approach aims to solve problems that are beyond what a single research group could handle.

\subsubsection{Predicting the Non-specific Binding of Clinical Imaging Agents}

Novartis established a consortium a few years ago to better understand how to optimize the properties of imaging agents. One of its aims was to determine which in vitro assay most precisely predicts the non-specific binding of imaging agents in the brain of human patients. This parameter is essential for clinical PET tracer development. It directly influences the quality of the images, which can only be judged after much work has been invested in developing a potential imaging candidate. An in vitro assay that could predict non-specific binding early would be highly informative.

Each party provided reference compounds to the other participants, as well as the results of their own assays. The combined expertise provided the basis for a thorough analysis, which no one could have conducted alone. Ultimately, this led to the conclusion that while all assays had intrinsic value, the measure of the chromatographic hydrophobicity index on immobilized artificial membranes $\left(\mathrm{CHI}_{\text {IAM }}\right)$ predicted the clinical performance of PET imaging agents most precisely and confirmed that it should be implemented early in the optimization process. ${ }^{[3]}$

\subsubsection{Swiss Industrial Biocatalysis Consortium}

Since its inception in 2004, the Swiss Industrial Biocatalysis Consortium (SIBC) ${ }^{[4]}$ has brought together scientists from the pharmaceutical, fine chemicals, agrochemical, flavour and fragrance industries, with the goal of promoting biocatalysis inside and outside of Switzerland, as well as facilitating pre-competitive knowledge sharing. The SIBC promotes biocatalysis by acting as consultants for industry and academia.

The 'Innovationsraum Biokatalyse' (innovation space biocatalysis) is an initiative with research and educational aspects which originated from Swiss universities, with the Zurcher Hochschule für Angewandte Wissenschafte (ZHAW) as the leading institution. Several members of the SIBC are serving on its scientific board and working groups, supporting the selection of scientific proposals for support. Among those selected was a joint proposal of Novartis and Syngenta with ZHAW, enabling common research on engineered halogenases, for the late-stage functionalization of valuable chemicals.

\subsection{Technology Development Projects}

With these collaborations, Novartis chemists seek to explore and develop novel technologies with academic partners, who provide expert knowledge and participate in a stimulating exchange of experience and ideas.

\subsubsection{Computer-assisted Synthetic Planning (CASP) for Biocatalysis}

The use of biocatalysis in synthesis is currently attracting growing interest in both the academic and industrial world. Over the last decade, the use of enzymes expanded significantly, and many new natural enzymes were characterized and made available. In addition, some enzymes were evolved artificially to enhance their selectivity and activity for well-established transformations such as reductions, oxidations, reductive aminations, esterifications and non-natural catalytic transformations (e.g. cyclopropanations). Enzymes are also increasingly utilized for transformations under non-natural conditions, such as under UV irradiation. 
The motivation to use enzymes in organic synthesis is driven by a need to simplify synthetic routes and to become more economical and selective compared to conventional syntheses. However, predicting whether a particular molecule might undergo a specific enzyme transformation is still a challenge. The continuously growing applications of enzymes in synthesis has increased the need to create an in silico 'expert system' to predict biotransformations. Professor Jean-Louis Reymond's group (Department of Chemistry and Biochemistry, University of Bern) has published several groundbreaking articles on machine learning applications in chemistry, which inspired Novartis research and development (R\&D) teams to propose a collaboration on this topic.

In this project, the team has exploited recent advances in computer assisted synthetic planning (CASP) using the 'molecular transformer'. The molecular transformer is a sequence-tosequence machine-learning model that can be trained to predict the products of organic transformations, including their stereochemistry, from the structure of reactants and reagents. Prof. Reymond and his team have used multi-task transfer learning to train the molecular transformer with one million reactions from the US Patent Office (USPTO) database (Fig. 4). This source of general chemistry knowledge was combined with 32'000 enzymatic transformations, each annotated with a text description of the enzyme. The group showed that the resulting Enzymatic Transformer model predicts the products formed from a given substrate and enzyme with remarkable accuracy, including typical kinetic resolution processes. Those very encouraging results are now being tested using a Novartis proprietary data set. ${ }^{[5]}$

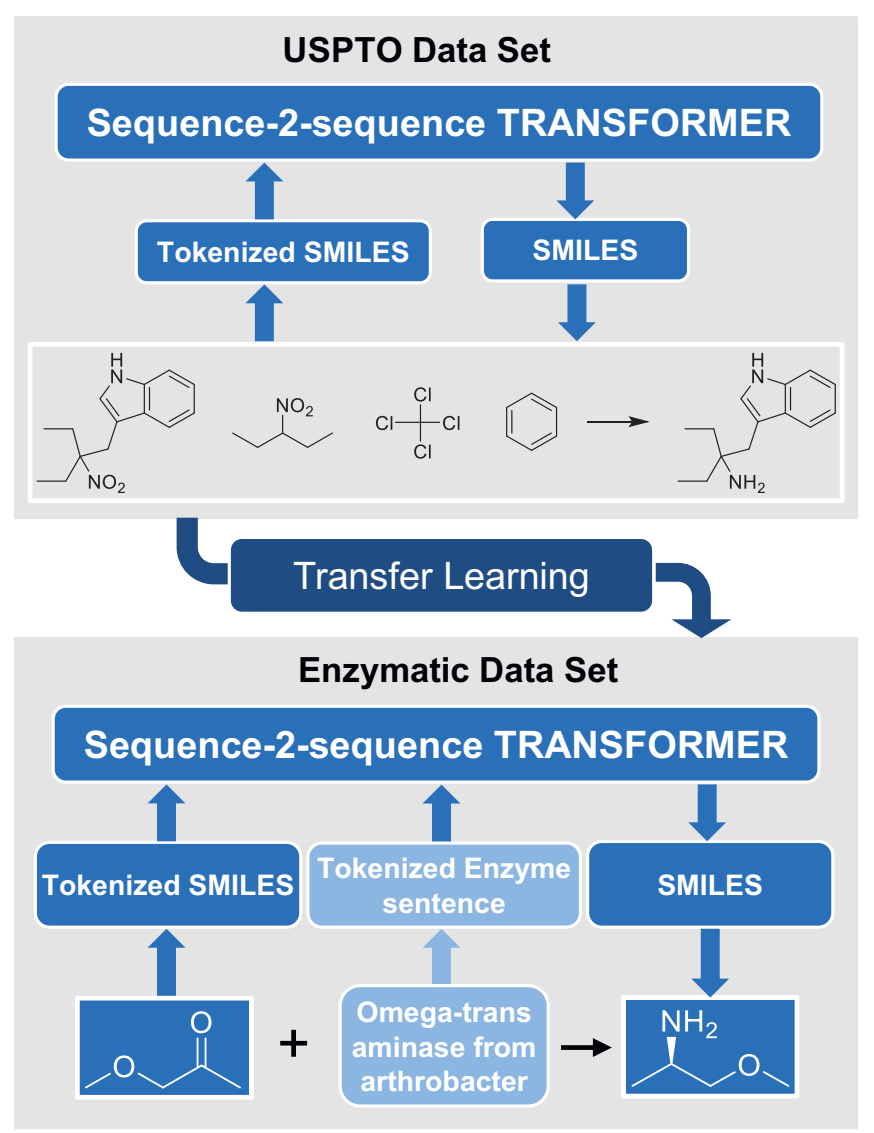

Fig. 4. General concept of the Enzymatic Transformer training.

\subsubsection{Automated Synthesis}

As automated synthesis emerges as a novel tool for medicinal chemistry, we are increasingly able to accelerate the design- make-test-analyze cycles of drug discovery using our automated workflows. In order to further evolve and rapidly bring the best molecules into our medicinal chemistry programs, we need to take our synthetic transformations to the next level. Prof. Jeffrey Bode and his group (ETH Zurich) are developing novel, cutting-edge chemistry that we are applying in the context of automated and plate-based synthesis. In collaboration with Prof. Bode, chemists at Novartis have developed workflows for the automated assembly of SLAP reagents and their photocatalytic conversion to substituted, saturated N-heterocycles (Fig. 5). The resulting libraries of morpholine and piperazine fragments were purified and subjected to a range of automated assays, providing a wealth of multi-parameter data describing their properties. We hope to learn whether this new chemical matter sits in a medicinal chemistry space with desirable properties, and to accurately predict the reaction outcome of this multi-step process.
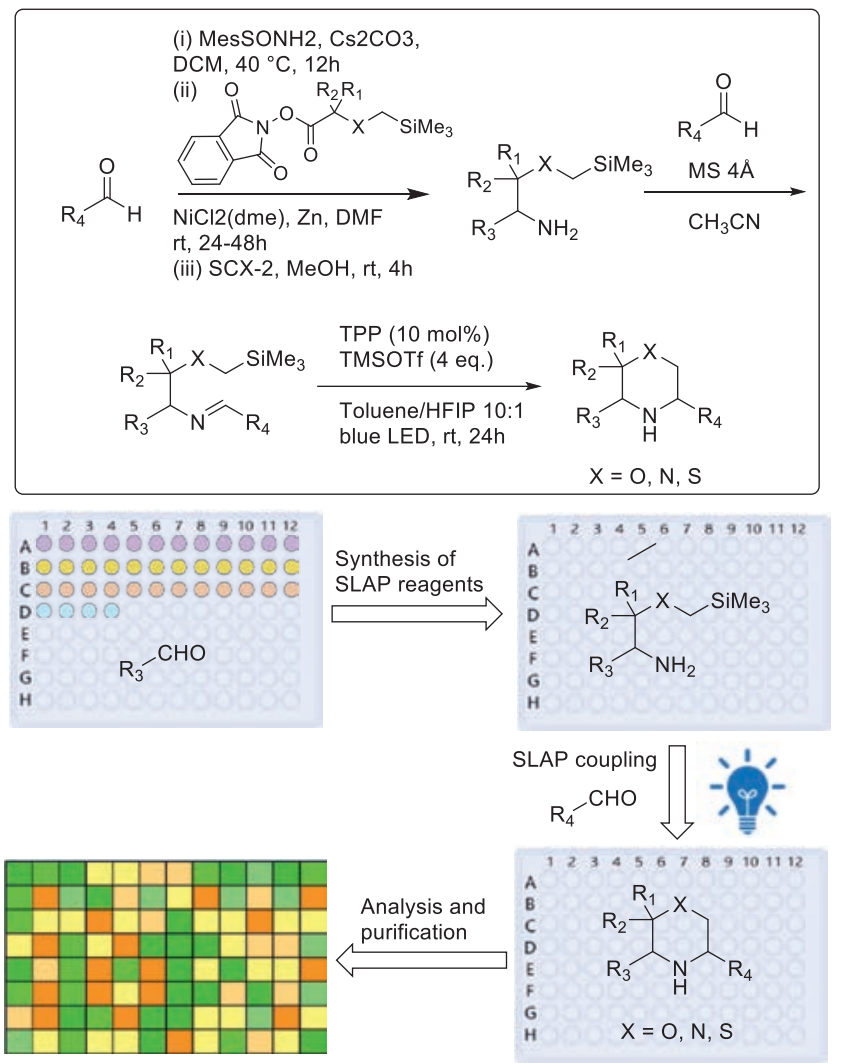

Fig. 5. Automated assembly of SLAP reagents and their photocatalytic conversion to substituted, saturated N-heterocycles.

\subsubsection{Novel Oxidative Enzymes for Late-stage Functionalization}

Scientific curiosity and a practical interest to expand oxidative enzymes beyond aromatic functionalization, as well as to selectively oxidize an unactivated aliphatic sp3 carbon center for production of high-value pharmaceuticals, led us to address this synthetic challenge with Prof. Rebecca Buller at ZHAW in Waedenswil. Innosuisse, the Swiss Innovation Agency, supported the project. During the course of this project, we modulated the chemoselectivity of a $\mathrm{Fe}(\mathrm{II}) / \alpha$-ketoglutarate-dependent dioxygenase for oxidative modification of unactivated aliphatic carbons of nonproteinogenic amino acids (Fig. 6). This was achieved by structure-guided enzyme evolution, during which the activity of the enzyme was increased as well.[6]

Interestingly, exchange of just one amino acid in the enzyme's active site (W40Y) led to a switch from natural hydrox- 
ylation activity to desaturation. This work holds the promise to further evolve this enzyme towards other non-native substrates, and a general utilization of this enzyme class for late-stage oxidative modifications and better scalability compared to what is achieved with P450-based enzymes.

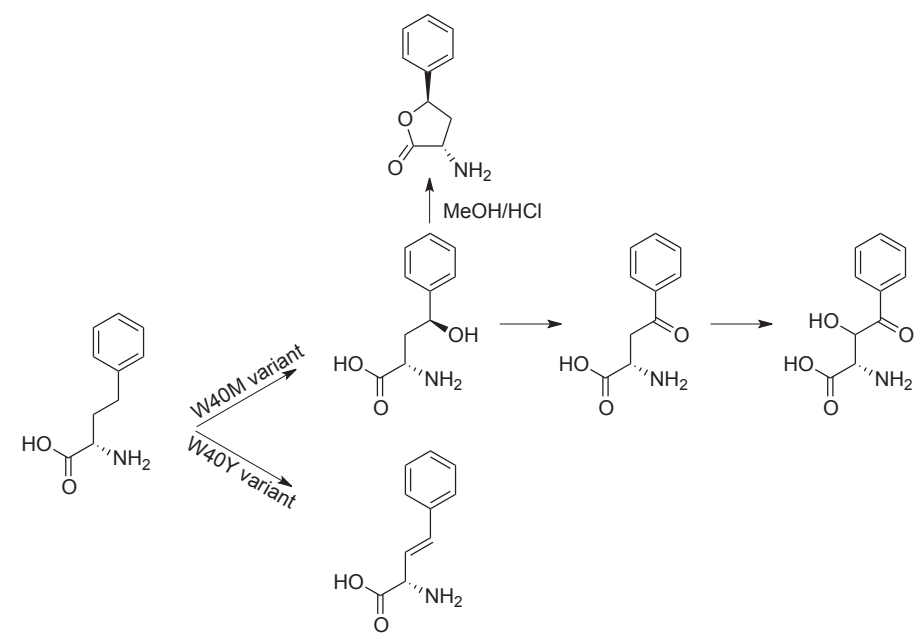

Fig. 6. Altered chemoselectivity of SmP4H enzyme variants, resulting in different L-homophenylalanine modification products.

\subsubsection{C-H Functionalization Methods}

$\mathrm{C}-\mathrm{H}$ functionalization reactions are complementary to conventional functional group-based reactivity. They expand the number of sites that can be targeted for derivatization, changing the way retrosynthesis is envisioned. Despite its obvious benefits in shortening a synthesis, the $\mathrm{C}-\mathrm{H}$ activation technology is still in its infancy and only few industrial applications have been reported. ${ }^{[7 a]}$ We initiated a collaborative project with Prof. Olivier Baudoin (University of Basel) to bridge the gap between academic research and the industrial use of $\mathrm{C}-\mathrm{H}$ functionalization technology. As a renowned expert in the field of $\mathrm{C}-\mathrm{sp} 3-\mathrm{H}$ functionalization, Prof. Baudoin was a natural partner to tackle a challenging $\mathrm{C}$-heteroatom bond formation reaction leading to amino-alcohol derivatives (Fig. 7).

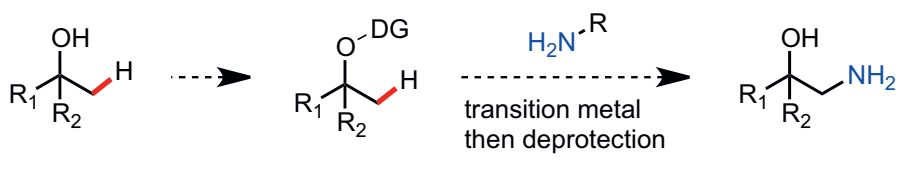

$$
D G=z_{3} \cdot N=<_{R}^{R}
$$

$>50$ examples 3rd alcohols: $53-92 \%$ 2nd alcohols: $30-99 \%$ methylene: $10-76 \%$

Fig. 7. $\mathrm{C}-\mathrm{N}$ bond formation reaction for the synthesis of amino-alcohol derivatives. DG: directing group.

The importance of such transformations is evident, as this motive is seen in many active pharmaceutical ingredients. Our collaboration started by defining how an ideal process of $\mathrm{C}-\mathrm{H}$ functionalization should look like when applied on scale, and to understand the prerequisites for such a reaction. Ultimately, we found adequate conditions to enable this reaction, providing the basis for a robust and efficient functionalization of various substrates. ${ }^{[7 b]}$

\subsubsection{Evaluating Alternative, Sustainable Solvents}

In the early 2000s, we became very interested in sustainable alternatives to the reprotoxic polar aprotic solvents, and into moving away from the petroleum feedstock. We started a variety of projects, one of them around the evaluation of sustainable alternative (also known as 'neoteric') solvents for classic nucleophilic aromatic substitution reactions. These typically require the use of reprotoxic, polar aprotic solvents such as dimethyl-formamide or $\mathrm{N}$-methyl-pyrrolidine. For this technical project, we partnered with the Institute of Chemical Technology at the Ecole d'Ingénieurs et d'Architectes de Fribourg. We were able to demonstrate that a variety of suitable options were available, requiring minor changes compared to the traditional way such transformations were run, for example using a different base or leaving group. ${ }^{\left[{ }^{[8}\right.}$ Applied to properly designed substrates, the reaction proceeded in a variety of sustainable solvents, some coming straight out of the bio feedstock (Fig. 8). More remarkable were the polar hindered esters, which proved highly effective and showed a limited extent of decomposition, if any. This came as a real eye-opener and encouraged us to more systematically challenge the use of established, toxic solvents.

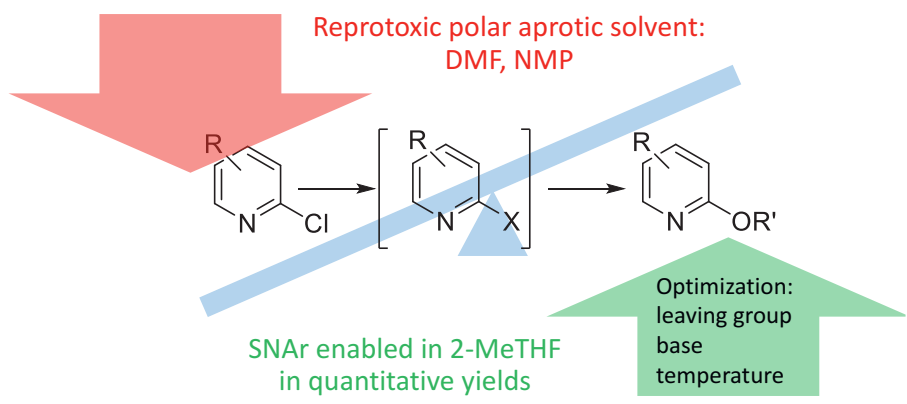

Fig. 8. SNAr on 2-chloropyridines, tipping the balance towards improved reaction conditions with sustainable solvents.

\subsubsection{Water-based Chemistry}

Over the last decades, the use of water as a solvent in organic synthesis attracted considerable attention, to improve sustainability, and to limit toxicity and safety issues. ${ }^{[9]}$ We have been working toward the adoption of the most socially responsible environmental standards for several years. ${ }^{[10]}$ To increase our impact in this endeavour, we triggered several research programs, including very successful collaborations with research partners in academia. ${ }^{[11]}$ Recently, a collaborative effort with the group of Prof. Christof Sparr (University of Basel) led to the development of a copper-free palladium-catalyzed Sonogashira cross-coupling reaction using bulky diadamantylalkylphosphanes (CataCXium) ligands and TPGS-750-M surfactant in water (Fig. 9). ${ }^{[12]}$ The facile and robust conditions co-developed in this joint effort enabled a highly selective, sustainable and very convenient access to a variety of propargylic systems.

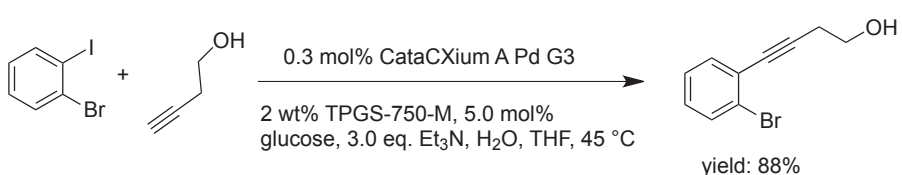

Fig. 9. Copper-free Sonogashira coupling in micellar medium.

The proximity of our research groups, and our common interest in sustainable synthetic methodologies facilitated the collaboration between Novartis scientists and the University of Basel. 
Students were invited to perform an internship within the Novartis R\&D labs, while Novartis chemists strongly interacted with the university. Our recent work with the Sparr group was done entirely at Novartis by a master student, who benefitted from a great training opportunity in a dynamic industrial environment, utilizing state-of-the-art technologies.

\subsubsection{Fluorination Chemistry}

Around 2005, we observed an increased use of fluorinated functionalities within our development portfolio and realized the need to complement our internal synthetic knowledge with external expertise. We triggered a variety of projects on this topic. Prof. Gilmour was at that time starting his independent career at the ETH Zurich and was identified as a partner for this purpose. We started by subsidizing a $\mathrm{PhD}$ thesis on fluorination chemistry for a young chemist. Within a few months, the research had led to remarkable findings on the inert steering effect of a 2-fluoro substituent on glycosylation reactions (Fig. 10). ${ }^{[13]}$ This initial success led us to rapidly intensify our interactions with our academic collaborator.<smiles>N=C(O[C@H]1O[C@H](COBr)[C@@H](OBr)[C@H](O)[C@H]1Br)C(Cl)(Cl)Cl</smiles>

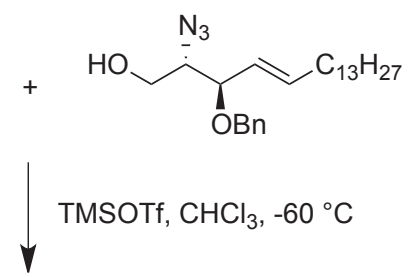

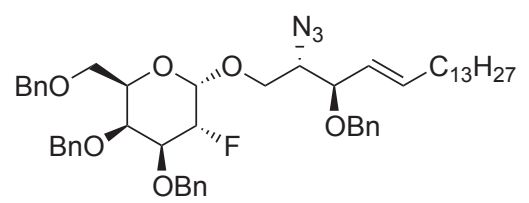

yield: $80 \%$ ratio a:b: $28: 1$

Fig. 10. Highly selective 2F-galactosylation.

Prof. Gilmour had published a method to open aziridinol with TBAF, to form the corresponding fluorinated oxazolidinone. A similar synthesis was envisioned for one of our drug candidates. The main challenge of this synthetic route was actually not the fluorination itself, for which Prof. Gilmour had encouraging precedents, but rather the enantio- and diastereoselective aziridination reaction of crotonaldehyde. We found a technical solution in the use of Jorgensen's organocatalyst, which allowed the formation of a chiral aziridinaldehyde (Fig. 11). The resulting product was directly reduced with $\mathrm{NaBH}_{4}$ to yield the alcohol with a reasonable diasteromeric ratio and good enantioselectivity. Treatment with TBAF led to the final product in $64 \%$ overall yield.

\subsection{Scientific Community Support}

Novartis actively supports science across the chemistry community. Our aspiration is to facilitate scientific exchange, encourage learning, collaboration and networking as well as generally support a community at the forefront of scientific progress, both in academia and industry.

\subsubsection{Support of Learned Societies}

Novartis chemists are directly involved in the activities of the Swiss Chemical Society (SCS) ${ }^{[14]}$ and of the European Federation for Medicinal chemistry and Chemical biology (EFMC). ${ }^{[15]}$ It provides financial support and participates in the initiatives, schools and congresses of both learned societies. Among others, Novartis chemists are involved in the annual meeting of the SCS and in
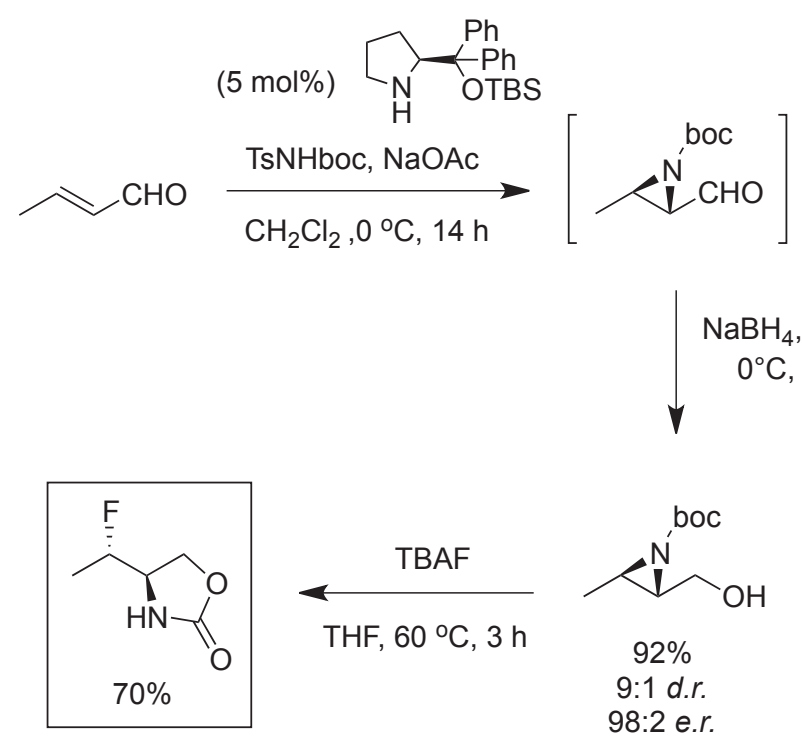

Fig. 11. Enantio- and diastereoselective aziridination reaction of crotonaldehyde.

the events of the Division of Medicinal Chemistry and Chemical Biology (DMCCB), ${ }^{[16]}$ such as the Swiss School of Medicinal Chemistry in Leysin. ${ }^{[17]}$ They are also involved in EFMC symposia including the International Symposium on Advances in Synthetic and Medicinal Chemistry (EFMC-ASMC), ${ }^{[18]}$ and the International Symposium on Medicinal Chemistry (EFMCISMC), ${ }^{[19]}$ In addition, Novartis supports initiatives for young investigators (EFMC's Young Scientists Network), ${ }^{[20]}$ and the Swiss Women in Chemistry initiative. ${ }^{[21]}$

\subsubsection{Facilitated Access to Screening Technologies (FAST)- Lab}

The FAST-Lab mediates interactions between Novartis and academic laboratories interested in identifying chemical compounds to modulate novel biology. It provides infrastructure (automated high-throughput screening and access to diverse non-proprietary compound libraries) as well as the expertise and mentorship that are required for academic laboratories to develop and conduct robust screening campaigns. The academic collaborator provides the hypothesis, the scientist to run the screening experiments in the FAST-Lab at Novartis and any specialized tools or reagents. Research plans implemented under the FAST-Lab model are "nostrings attached' agreements, where the collaborator is free to pursue the findings and has access to all structures of compounds emerging from the screen. Example of activities include the discovery of chemical probes for new targets, ${ }^{[22]}$ agents promoting cell differentiation ${ }^{[23]}$ and testing of new assay designs or screening technologies. ${ }^{\text {[24] }}$

A FAST-Lab agreement provides access to the NIBR Screening Set for External Collaborations, which consists of:

1. 50'000 non-proprietary compounds containing highly diverse LMW structures

2. 4'200 compounds with defined mode of action (MoA box ${ }^{[25]}$

3. $\sim 3000$ purified natural products

4. 160'000 additional compounds, available for structure activity expansion

The $50 \mathrm{~K}$ set contains a $\sim 7 \mathrm{~K}$ core set that can be used for assays with low throughput. Properties of compounds such as solubility and cell permeability were prioritized in addition to diversity, making this set attractive even for complex cellular assays. ${ }^{[26]}$

The FAST-Lab has completed more than 40 projects with 30 different institutions, at the two main Novartis research sites in Cambridge, USA and Basel, Switzerland. The selected collaborations fit to NIBR's general research interests, but do not directly 
overlap with any specific internal NIBR projects. Generally, they are exploratory and highly innovative, generating opportunities for both partners.

The FAST-Lab has had many interactions with Swiss Universities over its 5 years of existence. For instance, a collaboration with the Department of Biosystems Science and Engineering (DBSE) on a quorum sensing screen of the MoA box, resulting in the identification of 5-FU, an anticancer agent, which also blocks quorum sensing of biofilm-embedded methicillin-resistant Staphylococcus aureus (MRSA). 5-FU quenched the quorum-sensing activity as well in an in vivo model of implantassociated MRSA infections in mice, to such an extent that the infections could be completely cleared by the last-line antibiotic daptomycin, which otherwise achieves $<20 \%$ clearance rate when used alone.[27]

In conclusion, the FAST-Lab collaborations build trusted relationships between key academic partners and Novartis, based on shared scientific interests. Novartis shares its long-standing experience in compound screening, enabling academic groups to discover novel chemical tools and probes as they unravel novel biology or develop innovative technologies. In exchange, NIBR scientists have the opportunity to learn from the academic collaborators very early about the latest advances in areas of interest to the pursuit of innovative medicines.

\subsubsection{Internships at Novartis}

A recent survey ${ }^{[28]}$ highlighted that a significant portion of chemistry students wish to make a career in industrial R\&D. They also expressed a strong interest in internships within the industry, to gain insight into potential career paths. Novartis supports early career researchers from universities by offering several internship programs:

Basel Traineeship Program - A paid internship for 3 to 12 months, which may focus on therapeutic areas, discovery technologies, molecular pathway investigation, discovery chemistry or digital models. ${ }^{[29]}$ Students are given an innovative project to develop their scientific and practical knowledge, including feasibility studies and further development work that may lead to a scientific publication.

Post-Doctoral Program - Postdoctoral Fellows in research tackle fundamental scientific questions, with guidance from a Novartis investigator, as well as from an academic advisor, where appropriate. Projects are often inter-disciplinary in nature, bridging different disease areas or combining biology, chemistry and computational approaches. Discovery Fellows have the opportunity to publish their research in high-calibre peer-reviewed journals and present at international meetings. We encourage applications from candidates who wish to pursue a career either in academia or industry. ${ }^{[30]}$

Early Talent Program (ETP) - This 2-year program is a unique opportunity for recent $\mathrm{PhD}$ awardees to gain first experience in applied chemistry, process development, chemical manufacturing and analytical sciences or at the interface of bioorganic chemistry. Rotations within the Chemical Development Unit and pilot plant in Basel provides a comprehensive overview of drug development at Novartis. It enables ETP interns to transfer their theoretical expertise into practice and to establish a network within the industry. In contrast to what happens in standard post-doctoral setups, our ETP interns are directly involved in projects as part of a team, with defined responsibilities and support from experienced mentors. This program ensures that ETP interns are best prepared for their professional career. ${ }^{[31]}$

\subsubsection{FreeNovation}

Finally, and in addition to academic collaborations, the Novartis Forschungsstiftung promotes research via the FreeNovation ${ }^{[32]}$ funding program, which is open for academic investigators in
Switzerland. Its aim is to promote unusual and creative research, making funding available to support particularly original projects, free of legal restrictions. After the funding period, the investigators also have the opportunity to network with Novartis scientists while presenting their results at the FreeNovation Science Forum.

\section{Conclusions}

Scientific collaborations make sense when they serve mutual interests and benefit all partners. With this in mind, Novartis developed a range of collaboration opportunities in the area of medicinal chemistry and chemical development. In this setting, company chemists provide access to resources and knowledge. In return, they benefit from the expertise of the academic groups with whom they collaborate. While this article does not aim at exhaustively listing all scientific collaboration possibilities, it highlights the flexibility of our approach and our willingness to pursue research synergies with chemists in academia, as well as to support the extended chemistry community in Switzerland.

\section{Acknowledgements}

The authors would like to acknowledge all external partners with whom they had successful interactions over time, and in particular Professors Simon M. Ametamey, Roger Schibli and Linjing Mu (ETHZ), Ryan Gilmour (now in Muenster), Roger Marti (Fribourg), Christof Sparr (Basel), Olivier Baudoin (Basel), Jeffrey Bode (Zurich), Rebecca Buller (Waedenswil) and Jean-Louis Reymond (Bern), whose work is mentioned in the text, as well as Hans Widmer, who is in charge of the FreeNovation program at Novartis.

\section{Orcid numbers}

Arndt Meyer https://orcid.org/0000-0002-8845-9267; Daniel Baeschlin https://orcid.org/0000-0002-2227-7452; Cara E. Brocklehurst https://orcid.org/0000-0002-0484-5540; Fabrice Gallou https://orcid. org/0000-0001-8996-6079; Lucie E. Lovelle https://orcid.org/00000002-3373-0523; Michael Parmentier https://orcid.org/0000-00021732-9641; Radka Snajdrova https://orcid.org/0000-0002-4809-1066; Yves P. Auberson https://orcid.org/0000-0001-5800-4811

Received: August 13, 2021

[1] a) J. Streffer, V Treyer, A. Buck, S. M. Ametamey, M. Blagoev, R. P. Maguire, A. Gautier, Y. P. Auberson, M. E. Schmidt, I.-T. Vranesic, B. Gomez-Mancilla, F. Gasparini, NeuroImage 2021 230, 117785, https://doi.org/10.1016/j.neuroimage.2021.117785; $\quad$ b) A Deschwanden, B. Karolewicz, A.M. Feyissa, V. Treyer, S.M. Ametamey, A. Johayem, C. Burger, Y. P. Auberson, J. Sovago, C.A Stockmeier, A. Buck, G. Hasler, Am. J. Psychiatry 2011, 168, 727, https://doi.org/10.1176/appi.ajp.2011.09111607; c) S. Hintermann, I. Vranesic, H. Allgeier, A. Brülisauer, D. Hoyer, M. Lemaire, T. Moenius, S. Urwyler, S. Whitebread, F. Gasparini, Y. P. Auberson, Bioorg Med Chem 2007, 152, 903, https://doi.org/10.1016/j.bmc.2006.10.038.

[2] Y. He, D. M. Whitehead, E. Briard, S. Numao, L. Mu, R. Schibli, S. M. Ametamey, Y. P. Auberson, ChemMedchem 2020, 15, 2448, https://doi.org/10.1002/cmdc.202000340.

[3] L. Gobbi, J. Mercier, B. Bang-Andersen, J.-M. Nicolas, J. Reilly, B. Wagner, D. Whitehead, E. Briard, R. P. Maguire, E. Borroni, Y. P. Auberson, ChemMedChem 2020, 15, 585, https://doi.org/10.1002/cmdc.201900608.

[4] B. Wirz, M. Kittelmann, H.-P. Meyer, R. Wohlgemuth, Chimia 2010, 64 , 780, https://doi.org/10.2533/chimia.2010.780.

[5] D. Kreutter, P. Schwaller, J.-L. Reymond, chemrxiv 2020 , https://doi.org/10.26434/chemrxiv.13161359.v2.

[6] F. Meyer, R. Frey, M. Ligibel, E. Sager, K. Schroer, R. Snajdrova, R. Buller, ACS Catal. 2021, 11, 6261, https://doi.org/10.1021/acscatal.1c00678.

[7] a) T. Dalton, T. Faber, F. Glorius, ACS Cent. Sci. 2021, 7, 245, https://doi.org/10.1021/acscentsci.0c01413; b) K. Antien, A. Geraci, M. Partmentier, O. Baudoin, Angew. Chem. Int. Ed. 2021, 60, 22948, https:// doi.org/10.1002/anie.202110019.

[8] R. Marti, D. Spichiger, Chimia 2013, 67, 648, https://doi.org/10.2533/chimia.2013.648. 
[9] R. A. Sheldon, Green Chem. 2017, 19, 18 , https://doi.org/10.1039/C6GC02157C.

[10] F. Gallou, Chimia 2020, 74, 538, https://doi.org/10.2533/chimia.2020.538.

[11] a) M. Cortes-Clerget, T. Yu, J. R. Kincaid, P. Walde, F. Gallou, B.H Lipshutz, Chem. Sci. 2021, 12, 4237, https://doi.org/10.1039/D0SC06000C; b) U. Duong, T. Ansari, S. Parmar, S. Sharma, P. Kozlowski, J. Jasinski, S. Plummer, F. Gallou, S. Handa, ACS Sustain. Chem. Eng. 2021, 9, 2854, https://doi.org/10.1021/acssuschemeng.0c08792; c) T.-Y. Yu, H. Pang, Y. Cao, F. Gallou, B. H. Lipshutz, Angew. Chem. Int. Ed. 2021, 60, 3708 , https://doi.org/10.1002/anie.202013017; d) M. P. Andersson, F. Gallou, P. Klumphu, B. S. Takale, B. H. Lipshutz, Chem. Eur. J. 2018, 24, 6778, https://doi.org/10.1002/chem.201705524.

[12] M. Jakobi, F. Gallou, C. Sparr, M. Parmentier, Helv. Chim. Acta 2019, 102, e1900024, https://doi.org/10.1002/hlca.201900024.

[13] E. Durantie, C. Bucher, R. Gilmour. Chem. Eur. J. 2012, 18, 8208, https://doi.org/10.1002/chem.201200468.

[14] www.scg.ch

[15] www.efmc.info

[16] scg.ch/component/page/medicinal-chemistry

[17] For the next edition, postponed to 2022, see www.scmc22.scg.ch

[18] The next edition will take place in Zagreb, Croatia, with the latest information available at www.efmc-asmc.org

[19] After a virtual edition in 2021, EFMC-ISMC will take place in Nice, in 2022. Latest information at www.efmc-ismc.org/

[20] www.efmc.info/ysn

[21] scg.ch/component/page/women-in-chemistry

[22] a) Y. Tahara, D. Auld, D. Ji, A. A. Beharry, A. M. Kietrys, D. L. Wilson, M. Jimenez, D. King, Z. Nguyen, Eric T. Kool, J. Am. Chem. Soc. 2018, 140, 2105, https://doi.org/10.1021/jacs.7b09316; b) A. Okesli-Armlovich, A. Gupta, M. Jimenez, D. Auld, Q. Liu, M. C. Bassik, C. Khosla, Bioorganic \& Med. Chem. Lett. 2019, 29, 2559, https://doi.org/10.1016/j.bmcl.2019.08.010; c) A. G. Reidenbach, M. F. Mesleh, D. Casalena, S. M Vallabh, J. L. Dahlin, A. J. Leed, A. I. Chan, D. L. Usanov, J. B. Yehl, C. T. Lemke, A. J. Campbell, R. N. Shah, O. K. Shrestha, J. R. Sacher, V. L. Rangel, J. A. Moroco, M. Sathappa, M. C. Nonato, K. T. Nguyen, S. K. Wright, D. R. Liu, F. F. Wagner, V. K. Kaushik, D. S. Auld, S. L. Schreiber, E. V. Minikel, J. Biol. Chem. 2020, 295, 13516 , https://doi.org/10.1074/jbc.RA120.014905; d) J. W. Bushman, K. A. Donovan, N. J. Schauer, X. Liu, W. Hu, A. C. Varca, S. J. Buhrlage, E. S. Fischer, Cell Chem. Biol. 2021, 28, 78, https://doi.org/10.1016/j.chembiol.2020.09.005
23] S. A. Khan, K-m Park, L. A. Fischer, C. Dong, T. Lungjangwa, M. Jimenez, D. Casalena, B. Chew, S. Dietmann, D. S. Auld, R. Jaenisch, T. W. Theunissen, Cell Reports 2021, 35, 109233 https://doi.org/10.1016/j.celrep.2021.109233.

[24] D. S. Auld, J. Narahari, P. Ho, D. Casalena, V. Nguyen, E. Cirbaite, D. Hughes, J. Daly, B. Webb, Biochemistry 2018, 58, 4700, https://doi.org/10.1021/acs.biochem.8b00290.

[25] S. M. Canham, Y. Wang, A. Cornett, D. S. Auld, D. K. Baeschlin, M. Patoor, P. R. Skaanderup, A. Honda, L. Llamas, G. Wendel, F. A. Mapa, P. Aspesi Jr., N. Labbé-Giguère, G. G. Gamber, D. S. Palacios, A. Schuffenhauer, Z. Deng, F. Nigsch, M. Frederiksen, S. M. Bushell, D. Rothman, R. K. Jain, H. Hemmerle, K. Briner, J. A. Porter, J. A. Tallarico, J. L. Jenkins, Cell Chem. Biol. 2020, 27, 1124, https://doi.org/10.1016/j.chembiol.2020.07.004.

[26] A. Schuffenhauer, N. Schneider, S. Hintermann, D. Auld, J. Blank, S Cotesta, C. Engeloch, N. Fechner, C. Gaul, J. Giovannoni, J. Jansen, J. Joslin, P. Krastel, E. Lounkine, J. Manchester, L. G. Monovich, A. P. Pelliccioli, M. Schwarze, M. D. Shultz, N. Stiefl, D. K. Baeschlin, J Med. Chem. 2020, 63, 14425, https://doi.org/10.1021/acs.jmedchem.0c01332.

[27] F. Sedlmayer, A-K. Woischnig, V. Unterreiner, F. Fuchs, D. K. Baeschlin, N. Khanna, M. Fussenegger, Nucleic Acids Res. 2021 Apr 15:gkab251. https:// doi.org/10.1093/nar/gkab251. Epub ahead of print. PMID: 33856484.

[28] R. Dubey, A. Gimpela, K. Zouboulisa, M. Böcker, Chimia 2020, 74, 737, https://doi.org/10.2533/chimia.2020.737. See also C. Woolston, Nature 2019, 575, 403, https://doi.org/10.1038/d41586-019-03459-7.

[29] More under www.novartis.com/careers/students-scholars

[30] More under www.novartis.com/our-science/postdoc-program

[31] www.novartis.ch/de/karriere/studierende-hochschulabsolventen/traineeprogramme

[32] www.novartis.ch/de/novartis-in-der-schweiz/medizin-neu-denken/forschungsfoerderung/freenovation

\section{License and Terms}

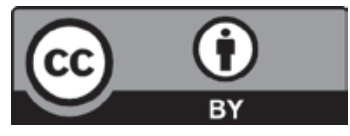

This is an Open Access article under the terms of the Creative Commons Attribution License CC BY 4.0. The material may not be used for commercial purposes.

The license is subject to the CHIMIA terms and conditions: (http:// chimia.ch/component/sppagebuilder/?view=page \&id=12).

The definitive version of this article is the electronic one that can be found at https://doi.org/10.2533/chimia.2021.936 